\title{
Stop-Frame Filming and Discovery of Reactions at the Single-Molecule Level by Transmission Electron Microscopy
}

Thomas W. Chamberlain, ${ }^{\dagger,}{ }^{\dagger}$ Johannes Biskupek, ${ }^{\S}$ Stephen T. Skowron, ${ }^{\dagger}$ Alexander V. Markevich, ${ }^{\dagger}$ Simon Kurasch, ${ }^{\S}$ Oliver Reimer, ${ }_{\nabla}$ Kate E. Walker, ${ }^{\dagger}$ Graham A. Rance, ${ }^{\dagger}$ Xinliang Feng, ${ }^{\perp}$ Klaus Müllen, ${ }^{\#}$ Andrey Turchanin, ${ }^{\nabla}$ Maria A. Lebedeva, ${ }^{\dagger}$ Alexander G. Majouga, ${ }^{\bigcirc}$ Valentin G. Nenajdenko, ${ }^{\circ}$ Ute Kaiser, ${ }^{*}$, Elena Besley, ${ }^{*}{ }^{\dagger}$ and Andrei N. Khlobystov* ${ }^{*}, \dagger$

${ }^{\dagger}$ School of Chemistry, University of Nottingham, Nottingham NG7 2RD, United Kingdom

${ }^{\ddagger}$ Institute of Process Research and Development, School of Chemistry, University of Leeds, Leeds LS2 9JT, United Kingdom

${ }^{\S}$ Central Facility of Electron Microscopy, Electron Microscopy Group of Materials Science, University of Ulm, 89081 Ulm, Germany

"Faculty of Physics, University of Bielefeld, 33615 Bielefeld, Germany

${ }^{\perp}$ Center for Advancing Electronics Dresden (cfaed) and Department of Chemistry and Food Chemistry, Technische Universitaet Dresden, 01069 Dresden, Germany

${ }^{\#}$ Max Planck Institute for Polymer Research, Ackermannweg 10, 55128 Mainz, Germany

${ }^{\nabla}$ Institute of Physical Chemistry, Friedrich Schiller University Jena, Lessingstraße 10, 07743 Jena, Germany

ODepartment of Chemistry, Moscow M.V. Lomonosov State University, Leninskiye Gory, Moscow 119992, Russia

Supporting Information

ABSTRACT: We report an approach, named chemTEM, to follow chemical transformations at the single-molecule level with the electron beam of a transmission electron microscope (TEM) applied as both a tunable source of energy and a sub-angstrom imaging probe. Deposited on graphene, disk-shaped perchlorocoronene molecules are precluded from intermolecular interactions. This allows monomolecular transformations to be studied at the single-molecule level in real time and reveals chlorine elimination and

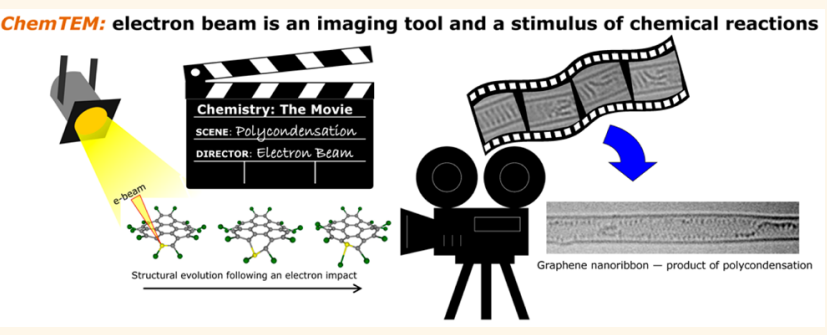
reactive aryne formation as a key initial stage of multistep reactions initiated by the $80 \mathrm{keV}$ e-beam. Under the same conditions, perchlorocoronene confined within a nanotube cavity, where the molecules are situated in very close proximity to each other, enables imaging of intermolecular reactions, starting with the Diels-Alder cycloaddition of a generated aryne, followed by rearrangement of the angular adduct to a planar polyaromatic structure and the formation of a perchlorinated zigzag nanoribbon of graphene as the final product. ChemTEM enables the entire process of polycondensation, including the formation of metastable intermediates, to be captured in a one-shot "movie". A molecule with a similar size and shape but with a different chemical composition, octathio $[8]$ circulene, under the same conditions undergoes another type of polycondensation via thiyl biradical generation and subsequent reaction leading to polythiophene nanoribbons with irregular edges incorporating bridging sulfur atoms. Graphene or carbon nanotubes supporting the individual molecules during chemTEM studies ensure that the elastic interactions of the molecules with the e-beam are the dominant forces that initiate and drive the reactions we image. Our $a b$ initio DFT calculations explicitly incorporating the e-beam in the theoretical model correlate with the chemTEM observations and give a mechanism for direct control not only of the type of the reaction but also of the reaction rate. Selection of the appropriate e-beam energy and control of the dose rate in chemTEM enabled imaging of reactions on a time frame commensurate with TEM image capture rates, revealing atomistic mechanisms of previously unknown processes.

KEYWORDS: transmission electron microscopy, carbon nanotube, graphene, single-molecule imaging, single-molecule reaction 
mechanism and are unable to rule out that an alternative mechanism may also result in the same macroscale observations. ${ }^{1,2}$ In a reaction mixture, molecules possess different amounts of kinetic energy (described by the Boltzmann distribution) and move chaotically, colliding with each other in random orientations, such that the intermolecular reaction is triggered only if the energy of the collision is sufficient to overcome the reaction activation energy. Commonly any inconsistencies between experimental observations and the proposed atomistic mechanism are mitigated simply by including empirical correlation parameters within the model, such as the steric factor which accounts for the hypothetical mutual orientation of the reacting molecules. ${ }^{1,2}$ However, in practice, definitive information about reaction mechanisms can only be provided by direct observation at the single-molecule level of the reactants transforming into products over time via metastable intermediates. This dream of every chemist has recently been brought closer to reality by a series of breakthroughs in scanning probe microscopy methods that can now shed light on the fundamental structure of individual molecules adsorbed on surfaces. ${ }^{3-9}$ In particular, noncontact atomic force microscopy (nc-AFM) has recently enabled imaging of the atomic structures of reaction products and reaction intermediates helping to decipher some complex organic reactions catalyzed by metal surfaces. ${ }^{3,9}$ The temporal resolution of scanning probe techniques is 10 orders of magnitude lower than the typical time scale of a chemical reaction. Therefore, the reactions on surfaces need to be activated by periodic heating (during which the molecules remain unobserved) and cooling cycles and thus still require statistical analysis of the distribution of different species. ${ }^{9}$ On the other hand, the latest advances in aberration-corrected high-resolution transmission electron microscopy (AC-HRTEM) have begun to provide an alternative way to study the mechanisms of chemical reactions. The combination of sub-angstrom spatial resolution with a larger overall field of view and an image capture rate significantly faster than scanning probe microscopy has enabled AC-HRTEM imaging of chemical transformations in low-dimensional continuous solids, such as Stone-Wales rearrangements, ${ }^{10}$ defect formation or metal atom entrapment in graphene, ${ }^{11-13}$ restructuring in carbon nanotubes, ${ }^{13-16}$ or transformations of inorganic nanostructures, ${ }^{17}$ as well as revealing fascinating molecular motion. ${ }^{18-20}$ These examples demonstrate that the TEM possesses a significantly greater bandwidth for temporal resolution than any other nanoscale imaging technique, with further improvement possible by combining carefully correlated laser beam and e-beam pulses in so-called 4D TEM. ${ }^{21}$ However, the outstanding time resolution of the 4D TEM technique has been achieved at the expense of spatial resolution, as 4D TEM is able to resolve features on the scale of tens of $\mathrm{nm}$ at best (i.e., 100 times bigger than atomic dimensions), thus precluding this method from imaging chemical reactions at the single-molecule level.

Up to now recording a "movie" of intermolecular reactions, where chemical transformations of a single, uniquely identifiable molecule are continually followed from the starting reactant all the way to the final product, via a series of intermediates, still remains a formidable challenge. In our chemTEM approach we utilize the e-beam of the microscope as both a fast imaging tool and a source of energy to stimulate chemical reactions. Such a dual-use of the e-beam enables us to trigger and record transformations of molecules as they happen, without the need to introduce any additional source of energy (such as heat in nc-AFM or laser pulses in 4D-TEM). ${ }^{3-9,21-23}$ Moreover, at different energies (accessible values range between $20-300 \mathrm{keV}$ ), the e-beam can trigger qualitatively different chemical processes in the same material, ${ }^{24}$ while tuning the e-beam dose rate may potentially control the rates of reactions. ${ }^{25}$ Therefore, our chemTEM methodology lends itself well to the recording of continuous "movies" of chemical reactions by acquiring a time series of AC-HRTEM images for a single molecule, where the reaction is taking place simultaneously with the imaging process.

The choice of molecules for chemTEM experiments is important: a candidate molecule should preferably have a well-defined symmetrical shape that could be easily identified in AC-HRTEM and have sufficient single-molecule contrast, and most importantly, the molecule must be unreactive until sufficient energy is delivered from the e-beam to break the bonds between the atoms and initiate reactions. Provided that the sample is very thin (i.e., a single molecule or a chain of molecules within a single-walled carbon nanotube (SWNT) or on a monolayer of graphene), it is the direct interactions of the e-beam with atomic nuclei that are the dominant forces, such that during AC-HRTEM imaging, the kinetic energy of fast electrons, controlled by the accelerating voltage of the microscope, is transferred directly to the atoms in ballistic collisions, with the maximum amount of energy received by a stationary atom in a collision being determined by the formula:

$$
T_{\max }=\frac{2 M E\left(E+2 m_{\mathrm{e}} c^{2}\right)}{\left(M+m_{\mathrm{e}}\right)^{2} c^{2}+2 M E}
$$

where $E$ is the energy of incident electrons ( $80 \mathrm{keV}$ in our case), $m_{\mathrm{e}}$ is the mass of an electron, $c$ is the speed of light and, most importantly, $T$ is inversely proportional to the atomic weight of the element $(M)$, so that under the same TEM imaging conditions, lighter elements receive significantly more energy from the e-beam. ${ }^{25}$ If the value of $T$ exceeds the energy threshold for atom displacement from the molecule $\left(E_{\mathrm{d}}\right)$, there is a nonzero likelihood for the molecule to become reactive as described by the cross section function $\sigma_{\mathrm{d}}$, the value which determines the rate of a given chemical transformation $(k)$ under the e-beam:

$$
k=j \cdot n \cdot \sigma_{\mathrm{d}}
$$

where $n$ is the number of bonds of this type in the molecule, and $j$ is the dose rate of the e-beam in units of $\mathrm{e}^{-} / \mathrm{nm}^{2} \cdot \mathrm{s}^{26}$ The latter parameter is precisely determined and readily controlled by TEM operating conditions which gives direct control of the rate of the observed reaction in chemTEM experiments: The greater the rate of the collisions between the fast electrons and the molecule, the faster the observed reaction. Therefore, provided that the molecule is stable at low dose rates of the e-beam of a given energy, the reactions of molecules can be readily accelerated or decelerated by adjusting the value of $j$ to make their rate commensurate with the TEM image capture. The overall effect of chemTEM is that the e-beam activates the molecules and drives their transformations, while taking a series of images of the processes which when reconstructed into a "movie" can create a continuous movement-a technique similar to stop-frame filming used in photography.

\section{RESULTS AND DISCUSSION}

In our study we investigate two simple molecules - perchlorocoronene (PCC) and octathio[8]circulene (OTC) - with disklike shapes easily distinguishable with a TEM. Having higher atomic numbers than carbon, $\mathrm{Cl}$ or $\mathrm{S}$ can provide a sufficient and clear contrast for single-molecule imaging, and the fact that $\mathrm{Cl}$ 
a
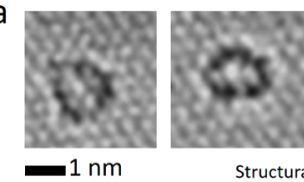

Structural evolution under the e-beam

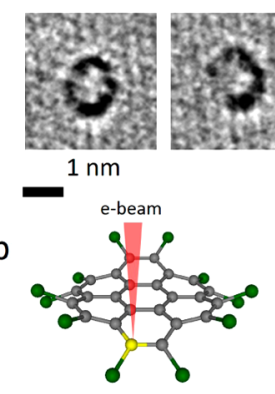

Structo

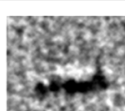

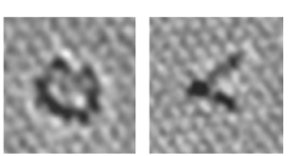
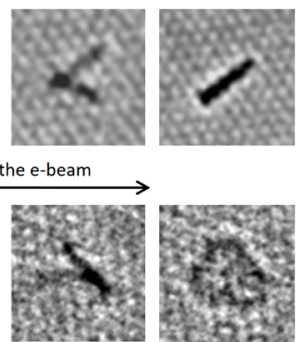

C

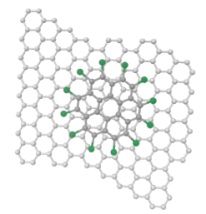

d

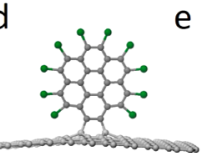

e

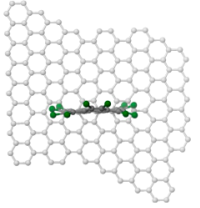

f
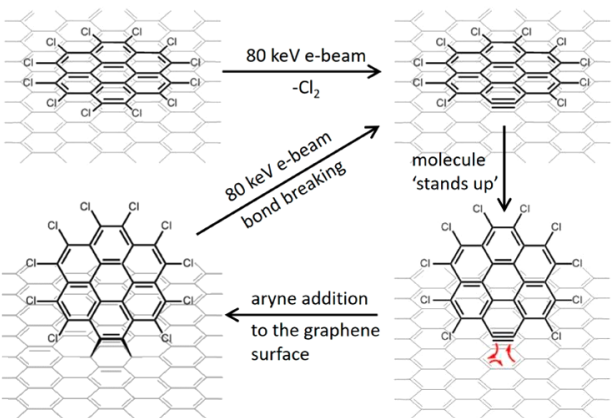

Figure 1. (a) Experimental AC-HRTEM images illustrating the transition of PCC from a face-on orientation (almost circular shape) to an edgeon orientation (line) on graphene observed under exposure to the $80 \mathrm{keV}$ electron beam (dose rate $\sim 10^{7} \mathrm{e} / \mathrm{nm}^{2} \cdot \mathrm{s}$; an interval of a few seconds exists between each image). Uneven contrast around the edge of the molecule (third and second images in the top and bottom time series, respectively) indicates the loss of Cl-atoms. (b) Snapshots from DFT calculations illustrating structural perturbations in PCC caused by energy transfer from the e-beam to a carbon atom (highlighted in yellow). Significant displacement of atoms in PCC from their equilibrium positions takes place, including the elongation of $\mathrm{C}-\mathrm{Cl}$ bonds up to $0.28 \mathrm{~nm}$ (equilibrium $\mathrm{C}-\mathrm{Cl}$ distance in PCC is $0.174 \mathrm{~nm}$ ). Structural diagrams showing PCC (c) lying face-on and (d, e) standing edge-on in two orthogonal orientations. (f) A proposed scheme of a monomolecular chemical reaction triggered by the $80 \mathrm{keV}$ electron beam: Energy transfer from the e-beam to a carbon atom breaks the $\mathrm{C}-\mathrm{Cl}$ bonds in PCC leading to aryne formation, which acts as a highly reactive dienophile and binds to the graphene covalently via Diels-Alder cycloaddition reaction. As a result of the cycloaddition to graphene, the molecule changes its orientation from face-on to edge-on. However, $\mathrm{C}-\mathrm{C}$ bonds between the aryne and graphene are stretched, and the graphene structure is deformed, which means that the adduct has only limited stability, as under further exposure to the e-beam, the bonds with graphene dissociate, and the molecule returns back to the face-on orientation (the aryne also appears to be able to switch from one edge-on orientation to another within a single frame, for example, frame 4 in (a), thus appearing as two lines at an acute angle). After this cycle of transformations, the PCC molecule becomes damaged (i.e., missing two $\mathrm{Cl}$ atoms) and thus activated for further transformations, changing its shape and orientation and becoming progressively fragmented by the e-beam (Supporting Video 1).

and $\mathrm{S}$ atoms are significantly heavier than carbon ensures higher stability of PCC and OTC under the e-beam as compared, for example, to the analogous hydrogen-containing molecules. ${ }^{25} \mathrm{We}$ employ single-layer graphene and SWNTs as substrates to support the molecules during their reaction because the low contrast of the atomically thin carbon structures enables virtually unobscured observation of individual molecules, while the extremely high thermal and electrical conductivities of graphene and SWNT effectively mitigate any ionization and heating effects of the e-beam on the molecules during chemTEM experiments. Our previous experimental and theoretical analyses of the behavior of molecules under the e-beam clearly indicate that it is elastic (knock-on) interactions with the e-beam that transfer the energy $T$ to the molecules which are dominant when a single molecule is adsorbed on graphene $e^{27,28}$ or in a carbon nanotube, ${ }^{25,29}$ which is different to complex radiolysis reactions previously documented for molecules in a crystal or in thick films under high energy e-beams (as in electron beam lithography), or to ionization processes taking place in molecular monolayers under a low energy e-beam. ${ }^{30}$ Overall, the kinetic energy of fast electrons in chemTEM experiments which is directly transferred to the atomic displacement within the molecule offers the most direct way of triggering and monitoring a chemical reaction which can be successfully interpreted within the framework of elastic interactions, as shown for the two types of molecules PCC and OTC discussed below.

The molecules deposited on graphene are in a face-on orientation with respect to the e-beam and are isolated from each other such that any reactions initiated by the e-beam will be monomolecular, whereas the confinement of the molecules within carbon nanotube brings them close together and is conducive to intermolecular reactions. Both of these approaches are potentially useful, and we studied PCC on graphene in order to explore the monomolecular process under e-beam irradiation before embarking on the more ambitious task of exploring intermolecular reactions within carbon nanotubes. Individual PCC molecules on a layer of graphene appear in AC-HRTEM images as dark, roughly circular shapes due to the higher contrast of the $\mathrm{Cl}$ atoms (Supporting Information, Figure S1). Experimental images of PCC on graphene appear to be significantly blurred as compared to the simulated images of the stationary molecules (Figure S1), indicating that the physisorbed molecules remain mobile under the applied imaging conditions (room temperature, high vacuum, image capture rate $\sim 1 \mathrm{~s}$ ); however the motion is not as fast as it could be expected for a mediumsized molecule on graphene, indicating that forces stronger than physisorption may exist between PCC and graphene under chemTEM conditions. Indeed, continuous monitoring of the behavior of a single PCC molecule by AC-HRTEM reveals that the same molecule can exist in two different orientations with respect to the graphene surface: the face-on (appears as a circle) and the edge-on (appears as a line, Figure 1a) with the latter being stable only for a few seconds before the molecules return back to the face-on orientation. These transitions are sudden and repeat numerous times on the time scale of several seconds, which can be clearly captured in the experimental AC-HRTEM video (Supporting Video 1), with the majority of edge-on oriented molecules aligned with the arm-chair direction of graphene (Figure 1a,f). The edge-on orientation appears to be able to switch from one direction to another within the time 


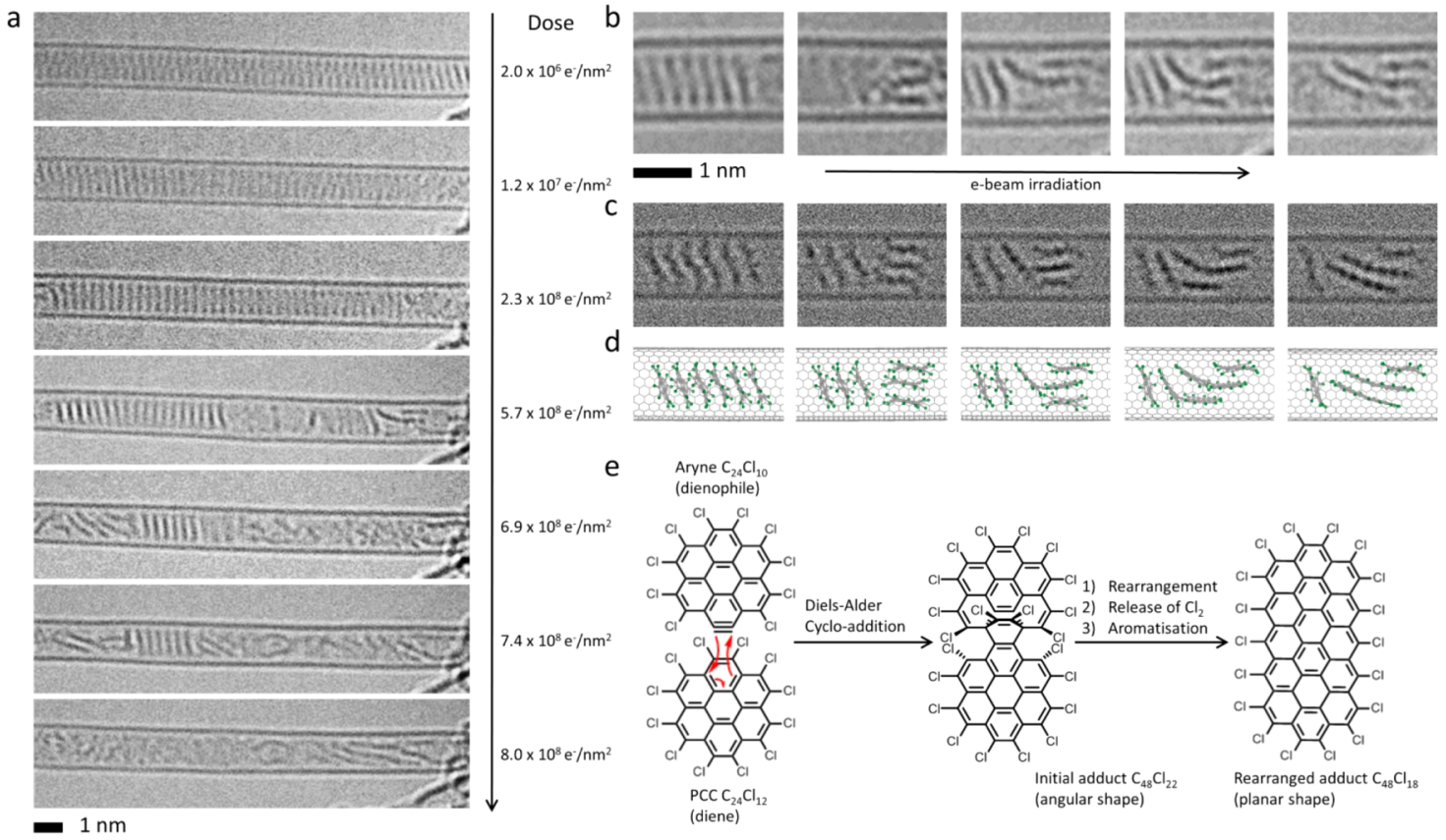

Figure 2. (a) Time-series AC-HRTEM images showing the transformation of a stack of PCC within a SWNT under $80 \mathrm{keV}$ e-beam exposure (the electron dose accumulated by the molecules during the time series is shown on the right side of each micrograph). (b-d) Close examination of the experimental images $(b)$ and comparison with simulated TEM images $(c)$, generated by exposing the corresponding structural models (d) to limited doses of $1 \times 10^{6} \mathrm{e}^{-} / \mathrm{nm}^{2}$ of $80 \mathrm{keV}$ electrons, indicate that intermolecular reactions are possible only when a PCC molecule can change its orientations with respect to the neighboring molecules: Two nonparallel molecules are able to join together to form an angular adduct which gradually transforms into a planar species approximately twice the length of the original PCC. (e) The intermolecular addition reaction observed by AC-HRTEM in carbon nanotubes is consistent with the Diels-Alder cycloaddition of an aryne (molecular formula $=\mathrm{C}_{24} \mathrm{Cl}_{10}$ ) to $\mathrm{PCC}$ predicted by DFT calculation and rearrangement of the initial adduct (angular) to an elongated flat polyaromatic molecule, $\mathrm{C}_{48} \mathrm{Cl}_{18}(\mathrm{planar})$, which continues reacting with further aryne species leading to the formation of ribbon-like structures terminated with chlorine atoms around the edge.

frame of a single image, effectively "walking" on the graphene, which results in two lines corresponding to the same molecule (for example, fourth frame in the time series, Figure 1a). Importantly, the shapes of the PCC molecules are no longer symmetric after such a transformation, indicating that the PCC has undergone some form of chemical modification due to an impact from the $80 \mathrm{keV}$ e-beam employed for AC-HRTEM measurements. A close inspection of experimental AC-HRTEM images indicates the loss of $\mathrm{Cl}$-atoms, manifested as a gap in the circle of dark contrast (Figure 1a, third and second images in the top and bottom time-series, respectively), suggesting chlorine elimination from PCC as a possible initial step.

The loss of chlorine atoms from the sterically strained PCC is explained by considering the elastic interactions of the e-beam with the atoms constituting the molecule. Thus, the $80 \mathrm{keV}$ e-beam in our experiments can transfer up to a maximum of $15.8 \mathrm{eV}$ of kinetic energy $T$ (eq 1 ) to a carbon atom and only $5.3 \mathrm{eV}$ to a chlorine atom of PCC. In both cases, the transferred energy causes displacement of the atoms from their equilibrium positions, but a detailed analysis of the PCC evolution postcollision with an $80 \mathrm{keV}$ electron by ab initio calculations predicts that the energy transfer from the $80 \mathrm{keV}$ e-beam to $\mathrm{Cl}$-atoms can cause only small perturbations in the molecule, not leading to any bond dissociation; however, the energy transfer to $\mathrm{C}$ atoms is large enough to trigger substantial structural changes, significantly stretching and thus activating the $\mathrm{C}-\mathrm{Cl}$ bonds (Figure 1b). Theoretical modeling of the PCC molecule under the $80 \mathrm{keV}$ electron beam (Supporting Video 2) combined with the experimentally observed structural changes suggest that graphene may act not only as a passive surface in AC-HRTEM experiments but also may play an additional role in promoting the dissociation of $\mathrm{C}-\mathrm{Cl}$ bonds due to interactions with the PCC molecules. While ballistic collisions between the fast electrons of the e-beam and atoms of the PCC molecule ${ }^{26}$ clearly provide the main source of energy explaining our experimental observations, any ionization of PCC, albeit not significant due to the highly conducting surface of graphene, could additionally reduce the energy barrier for dissociation of the $\mathrm{C}-\mathrm{Cl}$ bonds.

The observed abrupt transition of PCC from face-on to edge-on orientations is therefore consistent with dechlorinated PCC covalently bonding to graphene (Figure 1a,c-e), suggesting that an aryne of molecular formula $\mathrm{C}_{24} \mathrm{Cl}_{10}$, a product of dechlorination of PCC, can readily engage in bonding with the underlying graphene sheet via a cycloaddition reaction as predicted recently. ${ }^{31}$ Such a process demands a perpendicular (i.e., edge-on) orientation of $\mathrm{C}_{24} \mathrm{Cl}_{10}$ (Figure $1 \mathrm{f}$ ), closely matching our AC-HRTEM observations (Figure 1a). A theoretical study previously demonstrated that the bonding of arynes and other biradical species takes place preferentially along the armchair direction of the graphene sheet, while the addition of monoradicals or addition of biradicals along the zigzag direction leads to adducts with open electronic shell that are significantly less stable. ${ }^{32}$ This mode of bonding requires the aryne and graphene to act as a dienophile and a diene, respectively, leading to an adduct with a closed electronic shell (Figure 1f) which agrees well with the observed alignment of the edge-on structures with the armchair direction in graphene in our AC-HRTEM measurements (Figure 1a) and confirms that the sudden change of the orientation is driven by a chemical reaction rather than by a physical effect of the e-beam. While the Diels-Alder 


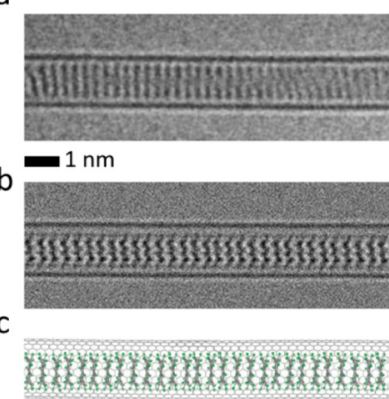

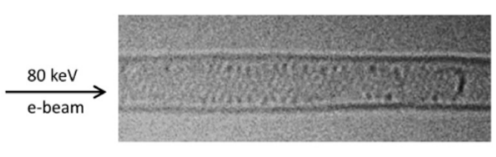
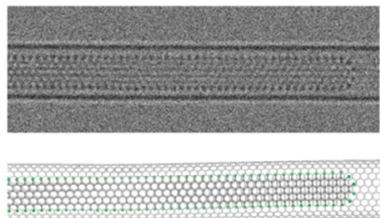

e
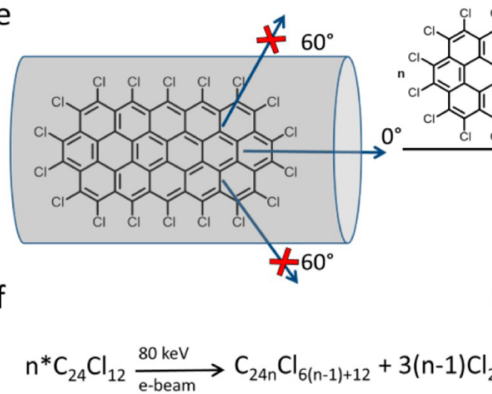

$\mathrm{h}$

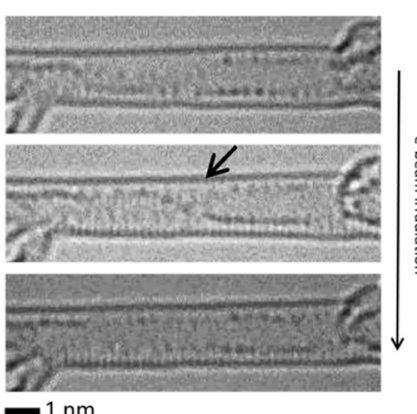

g $\quad \mid \| \leftarrow c$
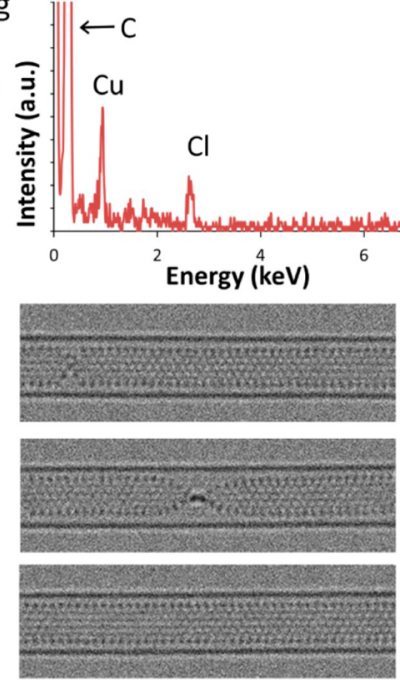

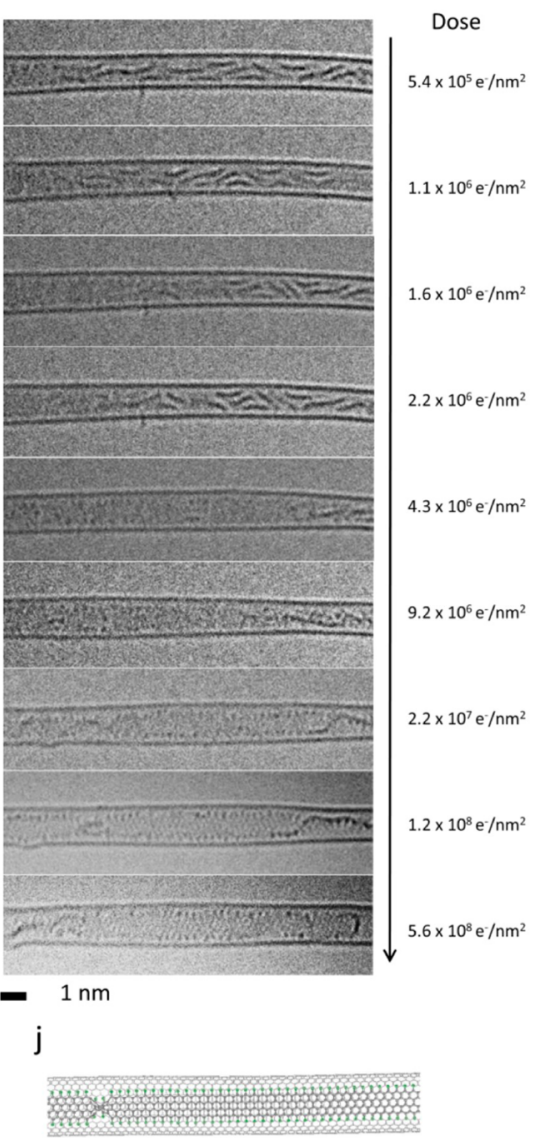

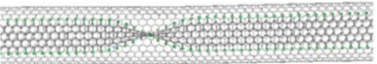

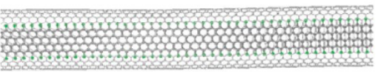

Figure 3. (a-c) HRTEM images (a), corresponding simulated TEM images (b), generated by exposing structural models (c) to a limited dose of $1 \times 10^{6} \mathrm{e}^{-} / \mathrm{nm}^{2}$ of $80 \mathrm{keV}$ electrons, showing the initial PCC@SWNT structure (left) and the resulting graphene nanoribbon terminated with chlorine atoms (right, Cl-atoms appear as dark dots along the edges of the nanoribbon) formed by e-beam-promoted elimination of chlorine atoms, aryne cycloaddition to PCC, rearrangement of the initial adduct to form polyaromatic molecules, and eventual polycondensation. (d) Experimental time-series images showing the intermediate steps of the transformation process from PCC oligomers to a continuous nanoribbon (the electron dose accumulated by the molecules during the time series is shown on the right side of each micrograph; note that because the molecules are already oligomerized at the start of this time series, the total electron dose required for nanoribbon formation is slightly lower than in Figure 2a which starts from intact PCC). (e) The carbon nanotube serves as a template for the reaction blocking all directions for aryne cycloaddition apart from the one parallel to the nanotube axis, which yields a strictly linear nanoribbon with the shape determined by the nanotube diameter. (f) Addition of each aryne increases the length of the nanoribbon by an additional $\left[\mathrm{C}_{24} \mathrm{Cl}_{6}\right]$ unit and releases three $\mathrm{Cl}_{2}$ molecules so that the overall reaction can be described as a polycondensation $(n \geq 2)$. Time-series AC-HRTEM images $(h)$ and corresponding simulated TEM images (i), generated by exposing the corresponding structural models (j) to a limited dose of $1 \times 10^{6} \mathrm{e}^{-} / \mathrm{nm}^{2}$ of $80 \mathrm{keV}$ electrons, illustrating rotation and twisting of the chlorinated graphene nanoribbon formed from PCC molecules inside the nanotube (a black arrow indicates the position of a twist in the nanoribbon). (g) Energy dispersive $\mathrm{X}$-ray spectrum confirming the presence of $\mathrm{Cl}$-atoms in the nanoribbon structure ( $\mathrm{Cu}$ peak is due to the specimen holder).

cycloaddition is clearly favorable for the stabilization of the reactive aryne species, it severely distorts the flat polyaromatic structure of the graphene making the adduct metastable, ${ }^{33,34}$ which means that under AC-HRTEM imaging conditions (high vacuum, room temperature, energy transferred from the e-beam to the atoms determined by eq 1 ), the aryne-graphene adduct is only transient. Further filming of PCC on graphene for extended periods of time indicates continuous fragmentation of PCC (e.g., progressive loss of chlorine and carbon atoms and extensive rearrangements) that do not lead to any stable products (Supporting Video 1). Therefore, beyond the crucial fact that aryne formation under the $80 \mathrm{keV}$ e-beam is the key step in activating the molecule for reactions, chemTEM imaging of monomolecular reactions has little relevance to intermolecular reactions that could potentially lead to stable and valuable products.

Knowing that PCC forms a highly reactive species, aryne, under the impact of the $80 \mathrm{keV}$ beam of electrons, we can now 


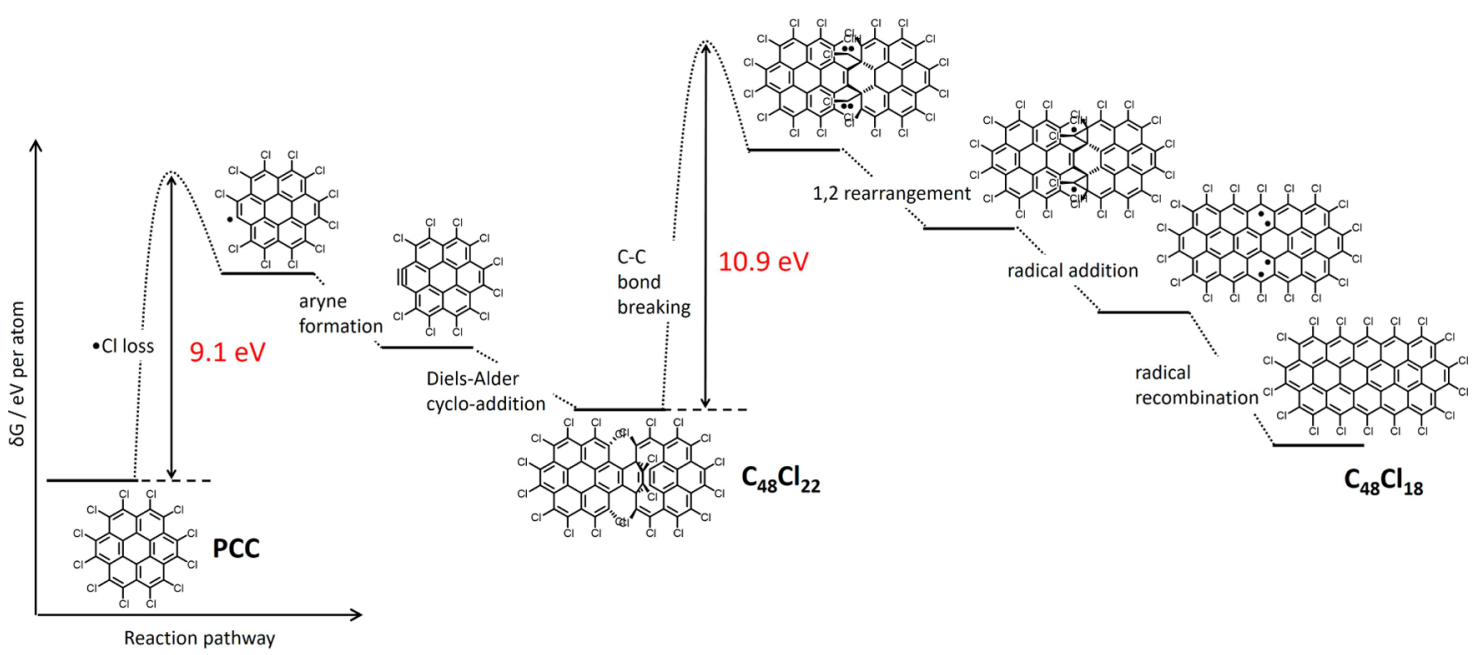

Figure 4. Energy profile of the intermolecular reactions observed for PCC in carbon nanotubes. Impact of the $80 \mathrm{keV}$ e-beam causes dissociation of $\mathrm{C}-\mathrm{Cl}$ bonds and the formation of a reactive aryne species (similar to the reaction of PCC observed on graphene). Provided that the space in the nanotube is sufficient for the aryne to reach a near-orthogonal orientation to a neighboring PCC molecule, the aryne (dienophile) and PCC (diene) undergo a Diels-Alder cycloaddition leading to the relatively stable $\mathrm{C}_{48} \mathrm{Cl}_{22}$ adduct. Under further e-beam irradiation, the initial adduct loses two $\mathrm{Cl}_{2}$ molecules and rearranges into a flat molecule $\mathrm{C}_{24} \mathrm{Cl}_{18}$ with an extended fully aromatic $\pi$-system.

generate the same aryne species inside a SWNT - a nanoscale reaction vessel where the close-packed molecules are predisposed for intermolecular reactions (Figure 2a). There is a significant advantage of the confinement imposed in the nanotube because, as compared to the molecules scattered on graphene or randomly moving in the gas or liquid phase: all reactant molecules in SWNT exist in the same initial state (i.e., all have the same kinetic energy and orientation), because they are strictly ordered and stacked in the SWNT (Figure 2a). Furthermore, entrapment in the nanotube can also decelerate chemical transformations due to the extreme spatial confinement restricting the movement of molecules, both individually preventing certain molecular conformations and, with respect to neighboring molecules, preventing sterically demanding orientations; therefore the SWNT provides ideal conditions for filming intermolecular reactions step-by-step, from the reactants all the way to the products.

PCC was inserted into SWNT with a mean diameter of $1.5 \mathrm{~nm}$ and confirmed by AC-HRTEM (Figure 2a,b top and left micrographs, respectively), where PCC is seen to exhibit a tendency similar to polyaromatic hydrocarbons to form stacks due to $\pi-\pi$ interactions, ${ }^{25,35-37}$ with each molecule separated solely by a van der Waals gap of $0.3-0.4 \mathrm{~nm}$ from its neighbors. Further confirmation was provided by Raman spectroscopy demonstrating a small red shift in the energy of the radial breathing modes (RBMs) of the filled relative to the empty nanotubes (Figures S13-S14), consistent with the confinement of guest species within the internal channel of a host nanotube effecting a softening of these vibrational modes. Despite the mutual proximity, PCC molecules entrapped in the nanotube (designated as PCC@SWNT) exhibit surprisingly low reactivity with each other, as our AC-HRTEM time series images show that intermolecular reactions occur only when a stack of PCC is disrupted (Figure 2a) (Supporting Video 3). Specifically, two molecules appear to join together to form an adduct twice the size of the original PCC, with an angle of $90-110^{\circ}$ between the two planes (Figure 2b, third and fourth frames). As the electron dose increases, the adduct undergoes planarization followed by further increases in length thus transforming the original PCC into a polymeric ribbon-like product with a width of $1.04 \mathrm{~nm}$
(Figure 2a,b, last frames). The edges of the nanoribbon are decorated with equidistantly positioned dark dots (Figure 3a,h), suggesting that atoms with a larger atomic number than carbon are terminating the edges of the structure. It is important to emphasize that the transformation of discrete PCC molecules to the polymeric nanoribbon, via a series of metastable inter mediates, has been recorded in a single experiment, continually acquiring frames of the reaction "movie" for the same molecules entrapped within the nanotube (Figure 2a, and Supporting Video 3), which means that the fate of each reacting molecule can be precisely traced throughout the entire multistep reaction process.

Under the $80 \mathrm{keV}$ e-beam, the molecules entrapped in a nanotube, PCC@SWNT, receive the same amount of energy from the fast electrons (eq 1) and experience similar conditions to those described earlier for PCC on graphene, so that the dechlorination process and the formation of reactive aryne species can be considered as an initial step in PCC@SWNT transformations. However, the molecular stacks persist visibly unchanged in the nanotube up to the point that a cumulative dose of $2 \times 10^{8} \mathrm{e} / \mathrm{nm}^{2}$ from the $80 \mathrm{keV}$ e-beam has been received by the sample, which is significantly greater than the theoretically predicted dose of $2.3 \times 10^{7} \mathrm{e} / \mathrm{nm}^{2}$ (Supporting Information, Section S2) that is required to trigger the dechlorination of PCC in the stacks. The discrepancy of a factor of 10 between the predicted and observed reactivities is explained by the unfavorable mutual orientations adopted by the molecules within the SWNT cavity and matches well our AC-HRTEM experimental observations where the neighboring PCC molecules appear to engage in intermolecular bonding only when the molecular stack loosens and creates more space for the molecules to adopt more favorable mutual orientations (Figure 2a,b). While dechlorination of PCC on graphene by the $80 \mathrm{keV}$ e-beam has been shown to lead to a Diels-Alder reaction of the resultant aryne with the underlying graphene sheet (Figure 1a), under the same experimental conditions, the aryne formed within SWNT will not be able to perform a cycloaddition with the concave side of the nanotube and therefore is forced to react with a neighboring molecule (Figure 2e). Since the Diels-Alder cycloaddition is a particularly sterically demanding process (e.g., the aryne on graphene must change to an edge-on orientation, Figure 1f), the 


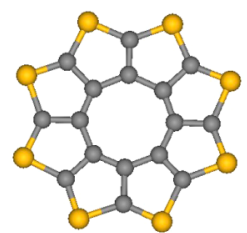

d

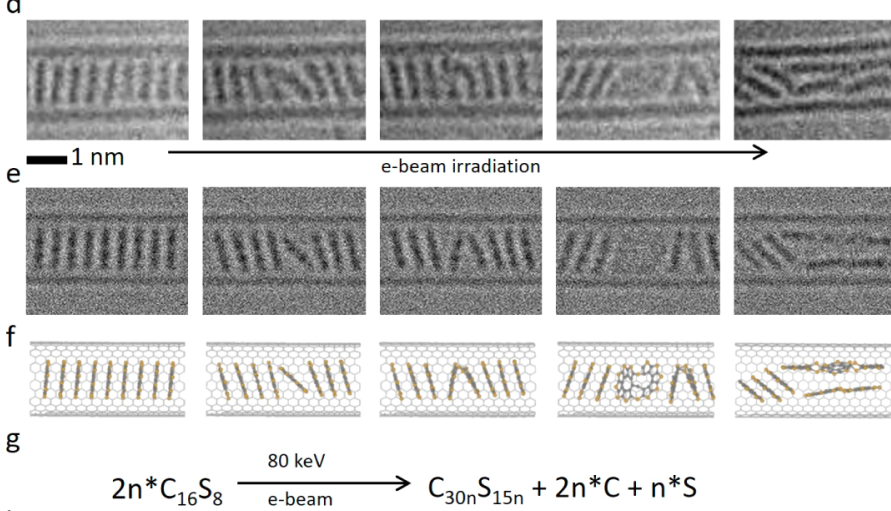

$\mathrm{h}$

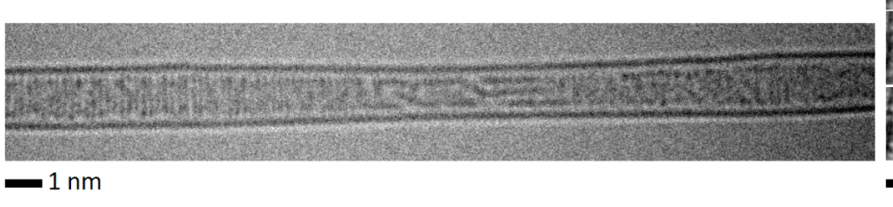

b

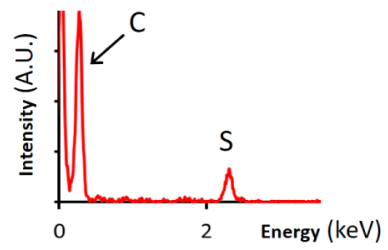

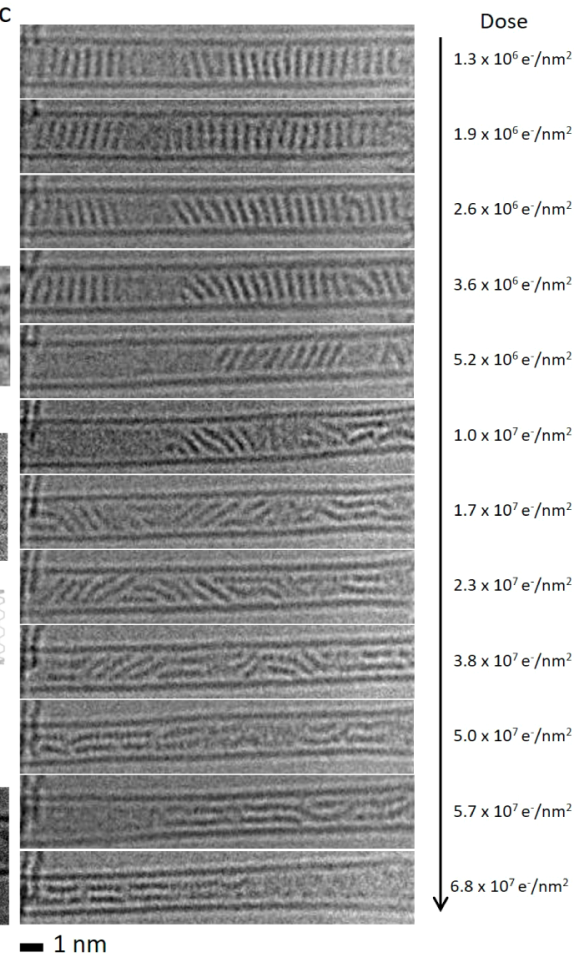

i

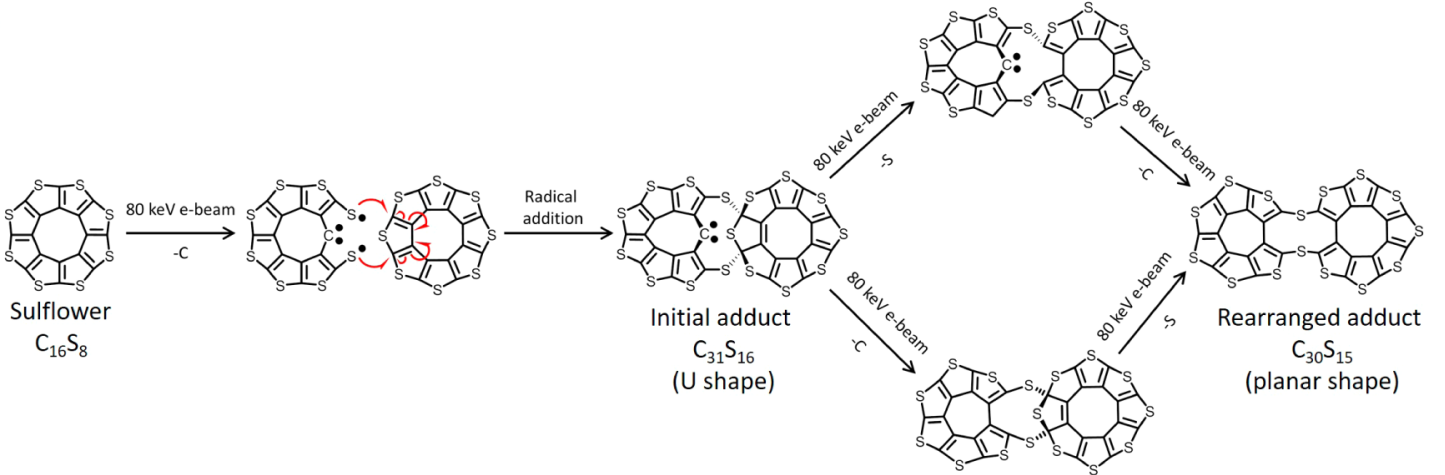

Figure 5. (a) Structural diagram of OTC. (b) EDX spectrum confirms the presence of sulfur in the OTC@SWNT structure. (c) AC-HRTEM timeseries images of OTC inside a SWNT undergoing polycondensation and the electron beam dose acquired by the molecules at each stage of the reaction. (d-f) Time-series AC-HRTEM images (d) of the initial reaction steps show good correlation with simulated TEM images (e), generated by exposing a structural model (f) to limited doses of $80 \mathrm{keV}$ electrons, and demonstrate the ability of OTC to react while still within the stack leading to U-shaped intermediate adducts which subsequently undergo planarization. $(\mathrm{g})$ Balanced reaction equation of the polycondensation taking place under AC-HRTEM conditions at $80 \mathrm{keV}$. (h) A polymeric product formed as a result of OTC polycondensation showing jagged irregular edges. (i) Pathways of reactions of OTC with neighboring molecules triggered by the 80 keV e-beam: Impact of the e-beam eliminates one of the $\mathrm{C}$ atoms, and the formed thiyl biradical attacks a neighboring OTC molecule leading to an angular $\mathrm{C}_{31} \mathrm{~S}_{16}$ adduct (U-shaped), which continues transforming via elimination of the sextet $\mathrm{C}$ atom and out-of-plane $\mathrm{S}$ atom (in either order, top and bottom pathways) to yield a planar $C_{30} S_{15}$ molecule. This process repeats with the $C_{30} S_{15}$ adduct reacting with a subsequent OTC molecule and ultimately leads to a ribbon-like product observed at the end of the AC-HRTEM experiment $(\mathrm{h})$.

reaction between the aryne and PCC can take place only if their planes adopt a particular angle of $c a \cdot 100^{\circ}$, which is not possible within the tight molecular stack in PCC@SWNT. Our theoretical modeling confirms that the angular adduct observed in the AC-HRTEM images corresponds to $\mathrm{C}_{48} \mathrm{Cl}_{22}$, which is a result of the Diels-Alder cycloaddition of an aryne $\mathrm{C}_{24} \mathrm{Cl}_{10}$ (dienophile) and PCC (diene), with an angular shape due to two bridging $\mathrm{sp}^{3}$-hybridized carbon atoms (Figure $2 \mathrm{~d}$ ), and is sufficiently long-lived to be captured by AC-HRTEM (Figure 2b).

In similar fashion to PCC on graphene, the kinetic energy of the e-beam transferred to an atom of PCC ( $T$, eq 1$)$ drives bond dissociation in the molecule within the SWNT, but because the bond dissociation energy threshold depends on the orientation of the bond with respect to the direction of momentum transferred from the e-beam, ${ }^{25}$ the energy thresholds for transformations of PCC inside nanotubes (in which the molecular plane is parallel to the e-beam) are expected to differ from those on graphene (molecular plane perpendicular to the e-beam). Our calculations predict two major energy barriers: $9.1 \mathrm{eV}$ to trigger the formation of the aryne from PCC and $10.9 \mathrm{eV}$ for the rearrangement of the angular Diels-Alder adduct (Figure 4). Both energy barriers can be overcome under our AC-HRTEM imaging conditions $(T=15.8 \mathrm{eV}$ for carbon, eq 1$)$. However, because the $10.9 \mathrm{eV}$ energy required to break the $\mathrm{C}=\mathrm{C}$ bridge in the Diels-Alder adduct, $\mathrm{C}_{48} \mathrm{Cl}_{22}$, is relatively high, only rare collisions with fast electrons of the e-beam can deliver sufficient 
energy to the molecule, which explains why the angular adduct $\mathrm{C}_{48} \mathrm{Cl}_{22}$ is kinetically stable enough to be observed in our experiments and transforms only gradually into flat structures corresponding to $\mathrm{C}_{48} \mathrm{Cl}_{18}$ via a 1,2-rearrangement (Figure 2e). The rearrangement process is driven by the e-beam initiated formation of carbon radicals within the structure which releases four chlorine atoms, and the resultant species reorganizes to form the flat molecule $\mathrm{C}_{48} \mathrm{Cl}_{18}$, gaining full aromaticity (Figure 4). This transformation proceeds via a series of metastable and hence transient intermediates which are too short-lived to be captured by AC-HRTEM. Overall, our AC-HRTEM imaging not only reveals the product of a multistep reaction, i.e., the polycondensation of PCC to form a nanoribbon, but also successfully captures an important intermediate, the angular Diels-Alder adduct, thus elucidating the two key steps of this complex chemical transformation: Diels-Alder addition and planarization of the adduct.

$\mathrm{C}=\mathrm{C}$ bonds within the planar species $\mathrm{C}_{48} \mathrm{Cl}_{18}$ (Figure $3 \mathrm{e}$ ) have similar properties to those in PCC and therefore can react with subsequent aryne molecules following an energy profile similar to that described above (Figure 4). The result of each aryne cycloaddition is that the molecule elongates by an additional $\left[\mathrm{C}_{24} \mathrm{Cl}_{6}\right]$ unit, and in the overall process of polycondensation, PCC molecules join the growing nanoribbon structure and release chlorine atoms (Figure $3 \mathrm{e}, \mathrm{f}$ ). It is important to emphasize that while the carbon nanotube does not participate in the transformations directly (unlike graphene), serving merely as a container, it has a significant effect on the reaction, e.g., defining the growth direction of the nanoribbon by blocking the nonlinear pathways of polycondensation (Figure 3e). The nanoribbon core has a structure identical to that of polyacene with a width of $1.04 \mathrm{~nm}$ (Figure $3 \mathrm{~h}-\mathrm{j}$ ) and equidistantly positioned Cl-atoms along the edges of the nanoribbons (chlorine atoms appear as dark dots, for example Figure $3 \mathrm{~h}$ ), clearly indicating that these are zigzag nanoribbon edges as opposed to an armchair edge in which the $\mathrm{Cl}$-atoms would be grouped in pairs. The estimated mass density of the nanoribbon@SWNT is about one-third lower than that of a PCC@SWNT stack (Supporting Information, Section S4), which may be the reason for the observed twisting of the nanoribbon (Figure $3 \mathrm{~d}, \mathrm{~h}$ and Supporting Information, Section S4) as it allows better compaction of the nanoribbon in the nanotube cavity. Because the nanotubes are very long (up to several micrometres) compared to the molecules, we do not observe their SWNT tips to confirm this experimentally. Graphene nanoribbons are materials with increasing importance for optical and electronic applications, as their functional properties can be adapted by strictly controlling their width, and there is a significant drive to develop efficient synthetic approaches for these materials. Continuous filming of intermolecular reactions in real space by AC-HRTEM in this study unveils an unexpected pathway of nanoribbon growth, previously unforeseen even hypothetically, and which we can now replicate on a preparative scale by heating PCC inside nanotubes at $700{ }^{\circ} \mathrm{C}$ in vacuum (Figure S15).

In order to demonstrate the generality of our approach, we also investigated octathio[8] circulene (OTC), a rare class of fully heterocyclic circulene molecule, ${ }^{38}$ which has a similar size and shape to PCC, but with the central disk of carbon atoms surrounded by eight sulfur atoms $\left(D_{8 h}\right.$ symmetry, Figure 5a). Because sulfur atoms can bridge between two carbon atoms, OTC is expected to exhibit reaction pathways markedly different to PCC under the same conditions. OTC was inserted into carbon nanotubes, as verified by the redshift in the position of the characteristic G band in the Raman spectrum of filled SWNT (Figures S14-S19), indicative of electron transfer from the electron-donating guest molecules to the electron-accepting host carbon nanostructure, and further investigated under the same conditions as PCC@SWNT. Entrapped in nanotubes, octathio[8]circulene forms stacks, OTC@SWNT (Figure 5c,d), that appear in AC-HRTEM images almost identical to PCC@SWNT, but time series imaging reveals that OTC molecules are more sensitive to the e-beam as the onset of their transformations takes place at a lower dose of the e-beam by a factor of 100 (Figure 5c). While neighboring PCC molecules require substantial reorientation within the nanotube to undergo chemical reactions, detailed analysis of OTC reaction videos (Supporting Video 4) and timeseries images (Figure 5c,d) surprisingly indicate that OTC molecules are able to react with each other while still essentially constrained within the stack, forming a U-shaped adduct with an interplanar angle of about $40^{\circ}$ (Figure $5 \mathrm{~d}$ ). Upon receiving further doses of electrons from the e-beam, the adduct of OTC undergoes planarization (Figure $5 \mathrm{c}$ ), thus gradually converting into a ribbon-like product inside the nanotube (Figure $5 \mathrm{c}$ bottom frame, and Figure $5 \mathrm{~h}$ ). The edge of the ribbon-like polymer has a higher contrast consistent with the presence of atoms with a higher atomic number than carbon along the edge (e.g., S atoms, confirmed by EDX, Figure 5b) and has a jagged appearance (Figure 5h) which differs significantly from the atomically smooth edge of the nanoribbon formed from PCC under the same conditions.

As in the case of PCC, detailed analysis of the e-beam interactions with OTC molecules sheds light on the nature of their transformations observed by AC-HRTEM. The energy transferred from the $80 \mathrm{keV}$ e-beam to the $\mathrm{C}$ atom of $15.8 \mathrm{eV}$ is significantly greater than that transferred to the $\mathrm{S}$ atom $5.9 \mathrm{eV}$ (eq 1 and Supporting Information, Section S3), which indicates that the chain of chemical transformations of OTC is triggered by e-beam impact on the $\mathrm{C}$ atom (Supporting Information, Section S5) in similar fashion as for PCC. Our theoretical calculations indicate that the elimination of a $\mathrm{C}$ atom from OTC leads to the formation of a reactive thiyl $\left(\mathrm{RS}^{\bullet}\right)$ radical species (Figures $5 \mathrm{i}$ and $\mathrm{S} 11$ ), which have very different steric requirements as compared to the aryne radicals derived from PCC under the same conditions. While the rigid planar $\mathrm{C}_{24} \mathrm{Cl}_{10}$ aryne must change its orientation within the nanotube in order to engage in a chemical reaction (Figure 2e), the $\mathrm{C}_{15} \mathrm{~S}_{8}$ thiyl radical is significantly more conformationally flexible and thus can attack the neighboring OTC via 1,4-addition to a thiophene group without any significant reorientation (Figure $5 \mathrm{i}$ ), leading to a U-shaped $\mathrm{C}_{31} \mathrm{~S}_{16}$ cycloadduct as shown by the AC-HRTEM time-series images (Figure $5 \mathrm{c}, \mathrm{d}$ ). The two flat aromatic parts in the $\mathrm{C}_{31} \mathrm{~S}_{16}$ cycloadduct bridged by sulfur and $\mathrm{sp}^{3}$-hybridized carbon atoms provide an interplane angle of $c a .40^{\circ}$ that is clearly observed in the initial stages of OTC@SWNT transformations under the $80 \mathrm{keV}$ beam (Figure $5 \mathrm{~d}$ and Supporting Video 3). Our DFT calculations confirm that the U-shaped $\mathrm{C}_{31} \mathrm{~S}_{16}$ cycloadduct is a stable species with a sextet carbon atom that exists as a Schrock-type carbene; however, AC-HRTEM imaging reveals that upon further exposure to the e-beam, it undergoes transformations leading to extended structures (Figure 5c) which are consistent with reaction pathways that involve the elimination of the $S$ atom of the former thiophene ring pushed out of the plane followed by elimination of the under-coordinated sextet $\mathrm{C}$ atom (Figure 5i, top), or these two processes in a reverse order (Figure $5 \mathrm{i}$, bottom), both leading to a planarized $\mathrm{C}_{30} \mathrm{~S}_{15}$ product in which two oligothiophene units are bridged by two 
sulfide bridges (Figure 5i), which can be clearly observed in AC-HRTEM time series (Figure $5 \mathrm{~d}$, last two frames). This process of radical addition, followed by $\mathrm{C}$ and $\mathrm{S}$-atom elimination, and finally planarization can repeat again at either end of the $\mathrm{C}_{30} \mathrm{~S}_{15}$ molecule, thus gradually converting the starting OTC compound to a ribbon-like polymeric product inside the nanotube (Figure 5c bottom frame and Figure 5h). AC-HRTEM images reveal that the polymer formed from OTC molecules has a complex undulating structure which cannot be described as either a solely zigzag or armchair edged nanoribbon because some sulfur atoms appear to have a role terminating the structure (in the thiophene ring) and while others act as bridging atoms (sulfide bridge) within the polymeric product (Figure S12), which is in stark contrast to the perfect polyacene (zigzag) nanoribbon formed from PCC under the same conditions. It is interesting that even after prolonged irradiation with the $80 \mathrm{keV}$ e-beam, we see no evidence for the formation of S-terminated nanoribbons with zigzag edges from OTC, which may be related to an insufficient amount of carbon in this molecule to form a continuous graphenic lattice containing nanoribbon as in the case of PCC, such that some $S$ atoms must be incorporated in the backbone of the polymeric product.

\section{CONCLUSIONS}

Our chemTEM approach is capable of continuously capturing images of intermolecular reactions, including the formation of transient reactive intermediates (aryne, Diels-Alder adduct, short polyaromatic intermediates, U-shaped and planar sulfidebridged oligothiophenes), in real space at the single-molecule level, imaging the entire pathway of the transformations of uniquely identifiable molecules, from initial reactants to the final products. The use of carbon nanostructures as supports for the molecules in our studies is crucial, as it ensures that the kinetic energy of fast electrons transferred directly to the atom is a dominant force causing atomic displacements, bond dissociations, and reactions, the rate of which is determined by the nature of the atom and the energy and dose rate of the e-beam. These are fully controlled by the operating conditions of the TEM and can be readily modeled using our theoretical framework developed for chem TEM. It is important to emphasize that transformations of the molecules are promoted by the e-beam itself, without any additional stimulus, and therefore the reactant molecules must be chosen such that they are activated only above a certain electron dose rate (eq 2) which allows moderation of the rates of the observed reactions to make them commensurate with the TEM image capture rate. For a multistep reaction, such as polycondensations of PCC or OTC, if the reaction intermediates are separated by sufficient energy barriers, their structures can also be imaged by ChemTEM, uncovering the reaction pathway at the molecular level. The comparison of two molecules with similar shapes but different composition, i.e., PCC and OTC, confined within a carbon nanotube cavity, demonstrates the significance of the steric factor in real space. The steric demands of the key reaction intermediates, the aryne and thiyl biradical formed from PCC and OTC, respectively, determine the reaction rate, with the latter being more reactive (by a factor of 100) due to the less strict steric requirements for reaction of the thiyl biradical. Significantly, we have demonstrated the potential of this approach not only to study known chemical reactions but also discover previously unknown reactions such as polycondensation, which leads to the formation of either chlorinated graphene nanoribbons or unusual polythiophenes, depending on the structure of the molecular precursor. The multistep mechanisms of these reactions determined by AC-HRTEM are complex and difficult to postulate a priori or from macroscopic observations, but our chemTEM time-series imaging provides the most direct method for enabling and studying chemical reactions at the molecular level.

\section{METHODS}

Computational Methods. The typical workflow for investigating possible electron beam (e-beam)-induced reactions of a molecule is as follows: An initial simulation of an electron impact is performed, corresponding to the largest possible transferral of energy $\left(T_{\max }\right)$ for the relevant beam energy and element and considering the angle of the electron beam with respect to the molecule. When repeated for all atoms of the molecule for which e-beam-induced structural changes are feasible, this identifies the molecule's susceptibility to direct knock-on (DKO) damage for the given beam energy. Taking the impacts that result in structural changes of interest, the impact simulations are repeated at lower energies until the minimum energy required to induce them (the threshold energy $E_{\mathrm{d}}$ ) is found to the desired accuracy (typically $0.1 \mathrm{eV}$ ). This can then be used to calculate the cross-section $(\sigma)$ of the reaction at any beam energy and therefore characterize the rate of this reaction for any combination of e-beam energy and electron dose rate $(j)$.

If the initial impact simulations are not exhaustive, they can be chosen based on experimental TEM observations. Meanwhile, the computationally optimized structures that result from this process can be used to aid image interpretation and structural analysis, particularly with the use of multislice TEM image simulations. An iterative "back-and-forth" process of comparison between the computational results, experimental TEM images, and proposed reaction steps can therefore be used to identify reaction mechanisms under the e-beam.

Threshold energies of beam-induced processes were calculated via density functional molecular dynamics (DFT-MD) simulations, performed as described in a previous study ${ }^{26}$ but using the B3LYP/6-31G* level of theory to accommodate for the increased electronegativity of the chlorine atoms; use of this basis set both quantitatively and qualitatively affected the results. The initial atomic velocities in the DFT-MD simulations were randomly sampled from a Maxwell-Boltzmann distribution at $298 \mathrm{~K}$. Reaction cross sections were calculated using these threshold energies (as an isotropic approximation) and the McKinleyFeshbach approximation, which enabled characteristic doses to be calculated in order to compare to the experimental TEM images. Thermal lattice contributions to cross sections were calculated from a MaxwellBoltzmann distribution at $298 \mathrm{~K}$.

Electron impacts are modeled using DFT-MD with a 1 fs time-step. The Q-Chem software package is used, ${ }^{39}$ utilizing a Fock matrix extrapolation procedure with a sixth-order polynomial and 12 Fock matrices in order to lower computational cost by using information from previous time-steps to accelerate SCF convergence times. ${ }^{40}$ The SCF convergence criterion is $10^{6}$, and the threshold for neglect of two electron integrals is $10^{9}$. The energy transferred from the incident electron to the nucleus is simply added to the existing velocity of the atom (due to molecular vibration) during a single time-step, as this interaction is extremely quick (on the order of approximately $10^{-22}$ seconds). The dynamics of the system are propagated on the Born-Oppenheimer surface until the reaction of interest has irreversibly occurred or until the energy is dissipated throughout the molecule and it is clear no further reaction will occur; this normally requires at most $500 \mathrm{fs}$ of simulation time and in most cases $<200 \mathrm{fs}$.

Materials Preparation. Perchlorocoronene (PCC) was prepared by treating coronene using conditions reported previously. ${ }^{41}$ IR spectroscopy and mass spectrometry confirmed the product formation, and the degree of chlorination was determined via GC-MS to be $>99 \%$. Octathio[8]circulene (OTC) was synthesized by a previously reported method. 38

SWNT (arc-discharge, P2-SWNT, Carbon Solutions Ltd.) were heated at $500{ }^{\circ} \mathrm{C}$ for $20 \mathrm{~min}$ in air. PCC $(15 \mathrm{mg})$ was mixed with the SWNT $(5 \mathrm{mg})$, sealed under vacuum $\left(10^{-6} \mathrm{mbar}\right)$ in a Pyrex glass ampule, and heated at $400^{\circ} \mathrm{C}$ for 3 days. The sample was allowed to cool 
and was then washed to remove excess molecules from the exterior of the SWNT by suspending in toluene $(20 \mathrm{~mL})$ with ultrasonication, followed by filtration through a PTFE membrane $(0.2 \mu \mathrm{m}$ pore size $)$ to give PCC@SWNT samples as a black powder. OTC was inserted into the SWNT from the gas phase following the same procedure as for PCC described above. The encapsulation of PCC and OTC in SWNT was additionally confirmed by EDX and Raman spectroscopy (Supporting Information, Section S8).

Graphene sheets grown by chemical vapor deposition of methane on copper foils were transferred onto TEM grids by procedures previously described. ${ }^{42}$ Deposition of PCC onto graphene was carried out by placing the solid compound in a Knudsen cell (Kentax Evaporator TCE-BSC), which was baked overnight at $200^{\circ} \mathrm{C}$ in ultrahigh vacuum. The temperature was further increased to $250{ }^{\circ} \mathrm{C}$, and after $30 \mathrm{~min}$ of thermal equilibration, the compound was evaporated for $30 \mathrm{~min}$ onto graphene. The adsorption of $\mathrm{C}_{24} \mathrm{Cl}_{12}$ on graphene was confirmed by in situ X-ray photoelectron spectroscopy (Multiprobe, Omicron) employing a monochromatic $\mathrm{Al} \mathrm{K}_{\alpha}$ radiation (Supporting Information, Section S9).

Transmission Electron Microscopy. The aberration-corrected HRTEM imaging was carried out using an image-side $\mathrm{C}_{\mathrm{s}}$-corrected FEI Titan 80-300 transmission electron microscope operated at $80 \mathrm{kV}$ acceleration voltage with modified filament extraction voltage to enhance the contrast and information limit. ${ }^{42}$ Images were recorded on a slow-scan CCD camera type Gatan Ultrascsan XP1000 with pixel binning by a factor of $2(1 \mathrm{k} \times 1 \mathrm{k}$ image pixel size $)$ using the prespecimen beam-blanker to avoid electron irradiation during the camera read-out time. Typical dose rates for AC-HRTEM imaging were between 0.5 and $2 \times 10^{6} \mathrm{e}-/ \mathrm{nm}^{2} / \mathrm{s}$ and exposure times between 0.5 and 1.0 s. $100 \mathrm{kV}$ imaging was performed on a JEOL2100 FEG TEM (used for initial assessment of the filling of nanotubes with PCC). For all in situ irradiation experiments, the microscope provided a highly controlled source of local and directed electron radiation on a selected area of the sample. The electron flux was kept to a minimum using low magnifications and short exposure times together with activated prespecimen blanker while searching for a suitable area, focusing and stigmating the lens, with the total search dose between 2 and $6 \times 10^{5} \mathrm{e}-/ \mathrm{nm}^{2}$. This corresponds to an accumulated dose typically used for 1 to 3 normal image acquisitions and does not contribute significantly $(<5-10 \%)$ to the total dose used for the observation of chemical transformations.

Subsequent TEM image simulations of structure models were performed using the multislice program QSTEM by C. T. Koch (http:// www.qstem.org). Image simulation parameters were $80 \mathrm{keV}$ electron energy, spherical aberration parameter $C_{\mathrm{S}}=10 \mu \mathrm{m}$, focus $=-8 \mathrm{~nm}$ (corresponds to Scherzer-focus condions with black atom contrast), focus spread $=4 \mathrm{~nm}$. The dampening effect of the camera (MTF, modulation transfer function) was included. The effect of limited electron dose was emulated by applying noise to the calculated (infinite dose) images using a custom-made Monte Carlo program exploiting the Poisson statistics of electrons. ${ }^{43}$

\section{ASSOCIATED CONTENT}

\section{S Supporting Information}

Additional information is available online as video files showing reactions of PCC on graphene and in carbon nanotubes, and a PDF containing The Supporting Information is available free of charge on the ACS Publications website at DOI: 10.1021/ acsnano.6b08228.

Details of calculations for molecules under the e-beam, additional micrographs showing PCC and OTC reactions in nanotubes and examples of products formed from these molecules, spectroscopy data (Raman, energy dispersive $\mathrm{X}$-ray analysis and X-ray photoelectron spectroscopy) and details of TEM image simulations (PDF)

Video S1: chemTEM video of reactions of PCC molecule on graphene under the $80 \mathrm{keV}$ e-beam (AVI)
Video S2: theoretical simulation of the $80 \mathrm{keV}$ e-beam impact on the outer carbon atom of PCC molecule causing displacements of the atoms from their equilibrium positions (MPG)

Video S3: chemTEM video of polycondensation of PCC inside carbon nanotube under the $80 \mathrm{keV}$ e-beam (AVI) Video S4: chemTEM video of polycondensation of OTC inside carbon nanotube under the $80 \mathrm{keV}$ e-beam (AVI) Video S5: chemTEM video of the latter stages of PCC polycondensation and formation of zigzag graphene nanoribbon terminated with $\mathrm{Cl}$-atoms under the $80 \mathrm{keV}$ e-beam (AVI)

Video S6: chemTEM video of the dynamic behavior of Cl-terminated graphene nanoribbon under the $80 \mathrm{keV}$ e-beam (AVI)

\section{AUTHOR INFORMATION}

\section{Corresponding Authors}

*E-mail: ute.kaiser@uni-ulm.de.

*E-mail: elena.besley@nottingham.ac.uk

*E-mail: andrei.khlobystov@nottingham.ac.uk.

ORCID

Stephen T. Skowron: 0000-0001-7322-5508

Klaus Müllen: 0000-0001-6630-8786

Valentin G. Nenajdenko: 0000-0001-9162-5169

Elena Besley: 0000-0002-9910-7603

Andrei N. Khlobystov: 0000-0001-7738-4098

Notes

The authors declare no competing financial interest.

\section{ACKNOWLEDGMENTS}

E.B., T.W.C., and A.N.K. acknowledge ERC Consolidator Grants and EPSRC for financial support and the Nanoscale and Microscale Research Centre (nmRC), University of Nottingham, for access to instrumentation. S.T.S., A.M., and E.B. are grateful to the High Performance Computing (HPC) Facility at the University of Nottingham for providing computational time. A.T., J.B., and U.K. acknowledge the support of the "Graphene Flagship" and DFG SPP "Graphene". U.K., J.B., and S.K. acknowledge the DFG and the Ministry of Science, Research and the Arts (MWK) of Baden-Wuerttemberg within the frame of the SALVE (sub-angstrom low-voltage electron microscopy) project. A.T. and O.R. acknowledge the DFG support through the grants (TU149/2-2 and TU149/5-1).

\section{REFERENCES}

(1) Atkins, P.; Jones, L. Chemical Principles: the Quest for Insight, 2nd ed.; W.H. Freeman \& Co: New York, 2002.

(2) Logan, S. R. Fundamentals of Chemical Kinetics; Longman Group Ltd.: London, 1996.

(3) De Oteyza, D. G.; Gorman, P.; Chen, Y.-C.; Wickenburg, S.; Riss, A.; Mowbray, D. J.; Etkin, G.; Pedramrazi, Z.; Tsai, H.-Z.; Rubio, A.; Crommie, M. F.; Fischer, F. R. Direct Imaging of Covalent Bond Structure in Single-Molecule Chemical Reactions. Science 2013, 340, 1434-1437.

(4) Di Giovannantonio, M.; El Garah, M.; Lipton-Duffin, J.; Meunier, V.; Cardenas, L.; Revurat, Y. F.; Cossaro, A.; Verdini, A.; Perepichka, D. F.; Rosei, F.; Contini, G. Insight into Organometallic Intermediate and its Evolution to Covalent Bonding in Surface-Confined Ullmann Polymerization. ACS Nano 2013, 7, 8190-8198.

(5) Hla, S.-W.; Bartels, L.; Meyer, G.; Rieder, K.-H. Inducing All Steps of a Chemical Reaction with the Scanning Tunnelling Microscope Tip: 
Towards Single Molecule Engineering. Phys. Rev. Lett. 2000, 85, 27772780.

(6) Hulsken, B.; Van Hameren, R.; Gerritsen, J. W.; Khoury, T.; Thordarson, P.; Crossley, M. J.; Rowan, A. E.; Nolte, R. J. M.; Elemans, J. A. A. W. Speller, S. Real-Time Single-Molecule Imaging of Oxidation Catalysis at a Liquid-Solid Interface. Nat. Nanotechnol. 2007, 2, 285289.

(7) Zhou, H.; Liu, J.; Du, S.; Zhang, L.; Li, G.; Zhang, Y.; Tang, B. Z.; Gao, H.-J. Direct Visualization of Surface-Assisted Two-Dimensional Diyne Polycyclotrimerization. J. Am. Chem. Soc. 2014, 136, 5567-5570.

(8) Treier, M.; Pignedoli, C. A.; Laino, T.; Rieger, R.; Müllen, K.; Passerone, D.; Fasel, R. Surface-Assisted Cyclodehydrogenation Provides a Synthetic Route Towards Easily Processable and Chemically Tailored Nanographenes. Nat. Chem. 2011, 3, 61-67.

(9) Riss, A.; Paz, A. P.; Wickenburg, S.; Tsai, H.-Z.; De Oteyza, D. G.; Bradley, A. J.; Ugeda, M. M.; Gorman, P.; Jung, H. S.; Crommie, M. F.; Rubio, A.; Fischer, F. R. Imaging Single-Molecule Reaction Intermediates Stabilized by Surface Dissipation and Entropy. Nat. Chem. 2016, 8, 678-683.

(10) Skowron, S. T.; Koroteev, V. O.; Baldoni, M.; Lopatin, S.; Zurutuza, A.; Chuvilin, A.; Besley, E. Reaction Kinetics of Bond Rotation in Graphene. Carbon 2016, 105, 176-182.

(11) Kotakoski, J.; Mangler, C.; Meyer, J. C. Imaging Atomic-Level Random Walk of a Point Defect in Graphene. Nat. Commun. 2014, 5, 3991.

(12) Robertson, A. W.; Lee, G. D.; He, K.; Yoon, E.; Kirkland, A. I.; Warner, J. H. Stability and Dynamics of the Tetravacancy in Graphene. Nano Lett. 2014, 14, 1634-1642.

(13) Rodriguez-Manzo, J. A.; Cretu, O.; Banhart, F. Trapping Metal Atoms in Vacancies of Carbon Nanotubes and Graphene. ACS Nano 2010, 4, 3422-3428.

(14) Rodriguez-Manzo, J. A.; Terrones, M.; Terrones, H.; Kroto, H. W.; Sun, L. T.; Banhart, F. In situ Nucleation of Carbon Nanotubes by Injection of Carbon Atoms into Metal Nanoparticles. Nat. Nanotechnol. 2007, 2, 307-311.

(15) Sun, L.; Banhart, F.; Krasheninnikov, A. V.; Rodriguez-Manzo, J. A.; Terrones, M.; Ajayan, P. M. Carbon Nanotubes as High-Pressure Cylinders and Nanoextruders. Science 2006, 312, 1199-1202.

(16) Chamberlain, T. W.; Meyer, J. C.; Biskupek, J.; Leschner, J.; Santana, A.; Besley, N. A.; Bichoutskaia, E.; Kaiser, U.; Khlobystov, A. N. Reactions of the Inner Surface of Carbon Nanotubes and Nanoprotrusion Process Imaged at the Atomic Scale. Nat. Chem. 2011, 3, 732-737.

(17) Sloan, J.; Matthewman, G.; Dyer-Smith, C.; Sung, A. Y.; Zheng, Z.; Suenaga, K.; Kirkland, A. I.; Flahaut, E. Direct Imaging of the Structure, Relaxation, and Sterically Constrained Motion of Encapsulated Tungsten Polyoxometalate Lindqvist Ions within Carbon Nanotubes. ACS Nano 2008, 2, 966-976.

(18) Koshino, M.; Niimi, Y.; Nakamura, E.; Kataura, H.; Okazaki, T.; Suenaga, K.; Iijima, S. Analysis of the Reactivity and Selectivity of Fullerene Dimerization Reactions at the Atomic Level. Nat. Chem. 2010, 2, 117-124.

(19) Harano, K.; Takenaga, S.; Okada, S.; Niimi, Y.; Yoshikai, N.; Isobe, H.; Suenaga, K.; Kataura, H.; Koshino, M.; Nakamura, E. Conformational Analysis of Single Perfluoroalkyl Chains by SingleMolecule Real-Time Transmission Electron Microscopic Imaging. J. Am. Chem. Soc. 2014, 136, 466-473.

(20) Khlobystov, A. N.; Porfyrakis, K.; Kanai, M.; Britz, D. A.; Ardavan, A.; Shinohara, H.; Dennis, T. J. S.; Briggs, G. A. D. Molecular Motion of Endohedral Fullerenes in Single-Walled Carbon Nanotubes. Angew. Chem., Int. Ed. 2004, 43, 1386-1389.

(21) Lorenz, U. J.; Zewail, A. H. Observing Liquid Flow in Nanotubes by 4D electron Microscopy. Science 2014, 344, 1496-1500.

(22) Zewail, A. H. Four-Dimensional Electron Microscopy. Science 2010, 328, 187-193.

(23) van der Veen, R. M.; Kwon, O.-H.; Tissot, A.; Hauser, A.; Zewail, A. H. Single-Nanoparticle Phase Transitions Visualised by FourDimensional Electron Microscopy. Nat. Chem. 2013, 5, 395-402.
(24) Zoberbier, T.; Chamberlain, T. W.; Biskupek, J.; Suyetin, M.; Majouga, A. G.; Besley, E.; Kaiser, U.; Khlobystov, A. N. Investigation of the Interactions and Bonding between Carbon and Group VIII Metals at the Atomic Scale. Small 2016, 12, 1649-1657.

(25) Chamberlain, T. W.; Biskupek, J.; Skowron, S. T.; Bayliss, P. A.; Bichoutskaia, E.; Kaiser, U.; Khlobystov, A. N. Isotope Substitution Extends the Lifetime of Organic Molecules in Transmission Electron Microscopy. Small 2015, 11, 622-629.

(26) Skowron, S. T.; Lebedeva, I. V.; Popov, A. M.; Bichoutskaia, E. Approaches to Modelling Irradiation-Induced Processes in Transmission Electron Microscopy. Nanoscale 2013, 5, 6677-6692.

(27) Chuvilin, A.; Kaiser, U.; Bichoutskaia, E.; Besley, N. A.; Khlobystov, A. N. Direct transformation of graphene to fullerene. Nat. Chem. 2010, 2, 450-453.

(28) Santana, A.; Zobelli, A.; Kotakoski, J.; Chuvilin, A.; Bichoutskaia, E. Inclusion of Radiation Damage Dynamics in High-Resolution Transmission Electron Microscopy Image Simulations: the Example of Graphene. Phys. Rev. B: Condens. Matter Mater. Phys. 2013, 87, 094110.

(29) Lebedeva, I. V.; Chamberlain, T. W.; Popov, A. M.; Knizhnik, A. A.; Zoberbier, T.; Biskupek, J.; Kaiser, U.; Khlobystov, A. N. The Atomistic Mechanism of Carbon Nanotube Cutting Catalyzed by Nickel under an Electron Beam. Nanoscale 2014, 6, 14877-14890.

(30) Turchanin, A.; Golzhauser, A. Carbon Nanomembranes: Carbon Nanomembranes. Adv. Mater. (Weinheim, Ger.) 2016, 28, 6075-6103.

(31) Sarkar, S.; Bekyarova, E.; Niyogi, S.; Haddon, R. C. Diels-Alder Chemistry of Graphite and Graphene: Graphene as Diene and Dienophile. J. Am. Chem. Soc. 2011, 133, 3324.

(32) Markevich, A.; Kurasch, S.; Lehtinen, O.; Reimer, O.; Feng, X.; Müllen, K.; Turchanin, A.; Khlobystov, A. N.; Kaiser, U.; Besley, E. Electron Beam Controlled Covalent Attachment of Small Organic Molecules to Graphene. Nanoscale 2016, 8, 2711-2719.

(33) Denis, P. A. Organic Chemistry of Graphene: the Diels-Alder Reaction. Chem. - Eur. J. 2013, 19, 15719-15725.

(34) Cao, Y.; Osuna, S.; Liang, Y.; Haddon, R. C.; Houk, K. N. DielsAlder Reactions of Graphene: Computational Predictions of Products and Sites of Reaction. J. Am. Chem. Soc. 2013, 135, 17643-17649.

(35) Okazaki, T.; Iizumi, Y.; Okubo, S.; Kataura, H.; Liu, Z.; Suenaga, K.; Tahara, Y.; Yudasaka, M.; Okada, S.; Iijima, S. Coaxially Stacked Coronene Columns Inside Single-Walled Carbon Nanotubes. Angew. Chem., Int. Ed. 2011, 50, 4853-4857.

(36) Fujihara, M.; Miyata, Y.; Kitaura, R.; Nishimura, Y.; Camacho, C.; Irle, S.; Iizumi, Y.; Okazaki, T.; Shinohara, H. Dimerization-Initiated Preferential Formation of Coronene-Based Graphene Nanoribbons in Carbon Nanotubes. J. Phys. Chem. C 2012, 116, 15141-15145.

(37) Botka, B.; Füstös, M. E.; Tóháti, H. M.; Németh, K.; Klupp, G.; Szekrényes, Z.; Kocsis, D.; Utczás, M.; Székely, E.; Váczi, T.; Tarczay, G.; Hackl, R.; Chamberlain, T. W.; Khlobystov, A. N.; Kamarás, K. Interactions and Chemical Transformations of Coronene Inside and Outside Carbon Nanotubes. Small 2014, 10, 1369-1378.

(38) Chernichenko, K. Yu.; Sumerin, V. V.; Shpanchenko, R. V.; Balenkova, E. S.; Nenajdenko, V. G. "Sulflower": A New Form of Carbon Sulfide. Angew. Chem., Int. Ed. 2006, 45, 7367-7370.

(39) Shao, Y.; Gan, Z.; Epifanovsky, E.; Gilbert, A. T. B.; Wormit, M.; Kussmann, J.; Lange, A. W.; Behn, A.; Deng, J.; Feng, X.; Ghosh, D.; Goldey, M.; Horn, P. R.; Jacobson, L. D.; Kaliman, I.; Khaliullin, R. Z.; Kuś, T.; Landau, A.; Liu, J.; Proynov, E. I.; et al. Advances in Molecular Quantum Chemistry Contained in the Q-Chem 4 Program Package. Mol. Phys. 2015, 113, 184-215.

(40) Herbert, J. M.; Head-Gordon, M. Accelerated, EnergyConserving Born-Oppenheimer Molecular Dynamics via Fock Matrix Extrapolation. Phys. Chem. Chem. Phys. 2005, 7, 3269-3275.

(41) Koshino, M.; Kurata, H.; Isoda, S. Study of Structures at the Boundary and Defects in Organic Thin Films of Perchlorocoronene by High-Resolution and Analytical Transmission Electron Microscopy. Ultramicroscopy 2010, 110, 1465-1474.

(42) Woszczyna, M.; Winter, A.; Grothe, M.; Willunat, A.; Wundrack, S.; Stosch, R.; Weimann, T.; Ahlers, F.; Turchanin, A. All-Carbon Vertical van der Waals Heterostructures: Non-Destructive Functional- 
ization of Graphene for Electronic Applications. Adv. Mater. (Weinheim, Ger.) 2014, 26, 4831-4837.

(43) Biskupek, J.; Hartel, P.; Haider, M.; Kaiser, U. Effects of Residual Aberrations Explored on Single-Walled Carbon Nanotubes. Ultramicroscopy 2012, 116, 1-7. 